\title{
Parâmetros na marcha na paralisia supranuclear progressiva: um estudo de caso
}

\author{
Gait parameters in progressive supranuclear palsy: a case study
}

\author{
Claudia Teixeira-Arroyo ${ }^{[\mathrm{a}]}$, Fabio Augusto Barbieri ${ }^{[\mathrm{b}]}$, Rodrigo Vitório ${ }^{[\mathrm{c}]}$, Marcelo Pinto Pereira ${ }^{[\mathrm{d}]}$, \\ Ellen Lirani-Silva ${ }^{[\mathrm{e},}$, Lilian Teresa Bucken Gobbi ${ }^{[f]}$
}

[a] Mestre em Ciências da Motricidade pela Universidade Estadual Paulista Júlio de Mesquita Filho (Unesp), membro do Programa de Atividade Física para Pacientes com Doença de Parkinson (PROPARKI) e do Laboratório de Estudos da Postura e da Locomoção (LEPLO), Universidade Estadual Paulista Júlio de Mesquita Filho (Unesp), Rio Claro, SP, docente do curso de Educação Física das Faculdades Integradas Fafibe, Bebedouro, SP - Brasil, e-mail: carroyo@rc.unesp.br

[b] Doutorando em Ciências da Motricidade pela Universidade Estadual Paulista Júlio de Mesquita Filho (Unesp), membro do Programa de Atividade Física para Pacientes com Doença de Parkinson (PROPARKI) e do Laboratório de Estudos da Postura e da Locomoção (LEPLO), Universidade Estadual Paulista Júlio de Mesquita Filho (Unesp), Rio Claro, SP - Brasil, e-mail: barbieri@rc.unesp.br

[c] Doutorando em Ciências da Motricidade pela Universidade Estadual Paulista Júlio de Mesquita Filho (Unesp), membro do Programa de Atividade Física para Pacientes com Doença de Parkinson (PROPARKI) e do Laboratório de Estudos da Postura e da Locomoção (LEPLO), Universidade Estadual Paulista Júlio de Mesquita Filho (Unesp), Rio Claro, SP - Brasil, e-mail: vitoriorodrigo@gmail.com

[d] Doutorando em Ciências da Motricidade pela Universidade Estadual Paulista Júlio de Mesquita Filho (Unesp), membro do Programa de Atividade Física para Pacientes com Doença de Parkinson (PROPARKI) e do Laboratório de Estudos da Postura e da Locomoção (LEPLO), Universidade Estadual Paulista Júlio de Mesquita Filho (Unesp), Rio Claro, SP - Brasil, e-mail: mppereir@yahoo.com.br

[e] Mestranda em Ciências da Motricidade pela Universidade Estadual Paulista Júlio de Mesquita Filho (Unesp), membro do Programa de Atividade Física para Pacientes com Doença de Parkinson (PROPARKI) e do Laboratório de Estudos da Postura e da Locomoção (LEPLO), Universidade Estadual Paulista Júlio de Mesquita Filho (Unesp), Rio Claro, SP - Brasil, e-mail: ellenls@rc.unesp.br

[f] Professora adjunta do Departamento de Educação Física do Instituto de Biociências da Universidade Estadual Paulista Júlio de Mesquita Filho (Unesp), coordenadora do Programa de Pós-Graduação em Ciências da Motricidade, do Programa de Atividade Física para Pacientes com Doença de Parkinson (PROPARKI) e do Laboratório de Estudos da Postura e da Locomoção (LEPLO), Rio Claro, SP - Brasil, e-mail: lilian.gobbi@pq.cnpq.br 


\section{Resumo}

Introdução: Comprometimentos na marcha de pacientes com paralisia supranuclear progressiva (PSP) podem aumentar o risco de quedas durante o andar, especialmente em ambientes complexos. Objetivo: Descrever o comportamento locomotor de uma paciente com PSP, nas condições de marcha livre e marcha adaptativa. Materiais e métodos: Estudo de caso de uma paciente com PSP (71 anos). Para análise cinemática, nas condições de marcha livre, com obstáculo baixo e alto, uma câmera digital registrou uma passada completa da paciente. Resultados: Com o aumento da complexidade do ambiente (marcha livre, obstáculo baixo e alto, respectivamente), foi observada diminuição do comprimento do passo $(0,37 \pm 0,07 ; 0,30 \pm 0,07$; $0,26 \pm 0,06 \mathrm{~m})$, do comprimento da passada $(0,71 \pm 0,11 ; 0,58 \pm 0,15 ; 0,47 \pm 0,07 \mathrm{~m})$ e da velocidade da passada $(0,55 \pm 0,14 ; 0,43 \pm 0,11 ; 0,36 \pm 0,11 \mathrm{~m} / \mathrm{s})$. Aumento progressivo ocorreu na duração do duplo suporte da passada livre $(29,47 \%)$ para a passada antes do obstáculo alto $(41,11 \%)$. Observou-se, ainda, ligeira diminuição na distância vertical pé/obstáculo alto (membro/abordagem: 7,18 \pm 1,88; e membro/suporte: $8,84 \pm 2,57 \mathrm{~cm}$ ) em relação ao obstáculo baixo (membro de abordagem: 8,86 $\pm 1,88$; e membro de suporte: $11,67 \pm 2,09 \mathrm{~cm})$. Conclusão: A PSP afetou de forma evidente a marcha da paciente. Inflexibilidade para a adaptação da marcha às demandas do ambiente foi observada durante a aproximação e a transposição dos obstáculos, o que pode aumentar o risco de tropeços e quedas.

Palavras-chave: Marcha. Paralisia supranuclear progressiva. Controle motor. Cinemática.

\section{Abstract}

Introduction: Gait impairments in patients with progressive supranuclear palsy (PSP) can lead to increased risk of trips and falls while walking, especially in complex environments. Objective: To describe the locomotor behavior of a woman with PSP during free and adaptive walking. Materials and methods: A case study of a PSP patient (71 years old). For kinematic data collection during free and adaptive walking (low and high obstacle crossing), a digital camcorder captured an entire gait cycle of the patient. Results: Decrease in step length $(0.37 \pm 0.07 ; 0.30 \pm 0.07 ; 0.26 \pm 0.06 \mathrm{~m})$, stride length $(0.71 \pm 0.11 ; 0.58 \pm 015 ; 0.47 \pm 0.07 \mathrm{~m})$, and stride velocity values $(0.55 \pm 0.14,0.43 \pm 0.11,0.36 \pm 0.11 \mathrm{~m} / \mathrm{s})$ was observed with the increasing of complexity (free walking, low obstacle crossing, and high obstacle crossing, respectively). The patient showed greater duration of double support phase in the high obstacle condition (41.11\%) than in the free walking condition (29.47\%). Also, toe clearance distance was slightly shorter in high obstacle condition (leading limb: $7.18 \pm 1.88$ and trailing limb: $8.84 \pm 2.57 \mathrm{~cm}$ ) than in the low obstacle condition (leading limb: 8.86 \pm 1.88 and trailing limb: $11.67 \pm 2.09 \mathrm{~cm}$ ). Conclusion: PSP clearly impairs the patient's gait. The patient showed inflexibility to adapt to environmental demands during both approaching and crossing obstacle. This behavior could increase the risk of trips and falls.

Keywords: Gait. Supranuclear progressive palsy. Motor control. Kinemathic.

\section{Introdução}

Depois da doença de Parkinson idiopática, a paralisia supranuclear progressiva (PSP) é a forma degenerativa mais comum de parkinsonismo, embora ainda seja considerada uma desordem neurológica rara (1). A prevalência de PSP no mundo é de aproximadamente 5/100.000 casos e incidência de 3 a 4 milhões por ano, com ligeira predominância em indivíduos do sexo masculino (2). 0 início da doença geralmente ocorre após os 60 anos, e a evolução é inexorável para o óbito em tempo variável de 1 a 17 anos, com sobrevida média de 6 anos $(2,3)$.

A PSP pode ser de causa idiopática ou vascular. Ambas as formas de apresentação revelam sinais e sintomas semelhantes, entretanto a forma idiopática se apresenta como parkinsonismo simétrico e sem evidências de outras etiologias; já na forma vascular, os sinais parkinsonianos são, em geral, assimétricos, com frequente incontinência esfincteriana e sinais de dano vascular nos exames de neuroimagem (4).

Independente da forma inicial de apresentação, estruturalmente a PSP é caracterizada por perdas 
neuronais, degeneração granulovascular, glioma e emaranhados neurofibrilares nos núcleos da base, núcleos cerebelares e tronco cerebral (2). Como consequência, a demência ocorre entre $60 \%$ e $80 \%$ dos casos $(1,5)$. Esquecimento, aparência apática e pensamento lento são frequentemente observados em pacientes com PSP (1). Os sinais e sintomas motores da doença incluem perda de equilíbrio, diminuição da mímica facial, comprometimento na fala, rigidez axial, instabilidade postural e paralisia do movimento dos olhos $(3,5)$.

A marcha também se apresenta comprometida na PSP. Os pacientes com PSP apresentam marcha em galope, anteroversão do tronco e hipometria, o que ocasiona quedas frequentes $(1,4,6)$. Apesar dos comprometimentos observados, não existe um padrão estabelecido em relação aos parâmetros da marcha em pacientes com PSP. Assim, há a necessidade de estabelecer esses parâmetros. Alterações nos parâmetros espaciais e temporais da marcha na doença de Parkinson em diferentes ambientes têm sido amplamente relatadas (7-9). Entretanto, a descrição dessas alterações em pacientes com PSP é escassa, especialmente em ambientes complexos.

Acredita-se que pacientes com PSP apresentem maior dificuldade para o controle prospectivo durante a locomoção em diferentes ambientes, devido às características motoras, visuais e cognitivas decorrentes da doença. Nesse sentido, objetiva-se, por este estudo, descrever o comportamento locomotor de uma paciente com PSP, nas condições de marcha livre e marcha adaptativa.

\section{Materiais e métodos}

Este estudo foi aprovado pelo comitê de ética local (processo 2722/2005). A paciente e o cuidador foram informados sobre os objetivos do estudo e assinaram o termo de consentimento livre e esclarecido, concordando em fazer parte desta pesquisa.

\section{Participante}

Participou deste estudo uma paciente, com diagnóstico de PSP. A participante é branca, natural da cidade de Rio Claro (SP), possui formação superior completa e é professora aposentada. Apresenta diagnóstico de PSP idiopática, sem histórico de outras doenças crônicas ou neurológicas associadas.
As características gerais da voluntária estão apresentadas no Quadro 1.

As características clínicas da paciente também foram avaliadas. Como a PSP é um tipo de parkinsonismo, escalas clínicas específicas para a doença de Parkinson - que identificam o estagiamento (escala de Hoehn and Yahr - H\&Y) e os comprometimentos (Unified Parkinson's Disease Rating Scale - UPDRS) da doença, nos domínios psíquicos (UPDRS I), funcionais (UPDRS II) e motores (UPDRS III) - foram aplicadas (10-12). Ainda, considerando as características típicas da PSP e o relato da paciente em relação à instabilidade, a Escala de Equilíbrio Funcional de Berg foi empregada (13), uma vez que o equilíbrio funcional é requisitado durante a realização das tarefas experimentais deste estudo. Para caracterizar as condições cognitivas da paciente, foram aplicados o Teste do Relógio, para avaliar a condição das funções executivas (14), e o Mini Exame do Estado Mental (MEEM), com o objetivo de fazer um rastreio de diferentes funções cognitivas da paciente (15). Todas as avaliações foram realizadas com a paciente no estado on da medicação (Quadro 2).

Quadro 1 - Características gerais da paciente

(Continua)

Características demográficas e antropométricas

\begin{tabular}{|c|c|}
\hline Idade (anos) & 71 \\
\hline Massa corporal (kg) & 79 \\
\hline Estatura (m) & 1,55 \\
\hline Escolaridade (anos) & 14 \\
\hline $\begin{array}{l}\text { Tempo de duração da doença } \\
\text { (anos) }\end{array}$ & 5 \\
\hline Quedas no último ano & 8 \\
\hline $\operatorname{AVD}(\%)^{\star}$ & 10 \\
\hline \multicolumn{2}{|c|}{ Laudo do exame de ressonância magnética } \\
\hline \multicolumn{2}{|c|}{$\begin{array}{l}\text { Atrofia leve da fissura Sylviana, região frontal, núcleos da base } \\
\text { e da região cerebelar. }\end{array}$} \\
\hline Tratamento medicamentoso & (mg/dia) \\
\hline Pramipexol & 4 \\
\hline Losartan & 50 \\
\hline Oxalato de escitalopram & 15 \\
\hline Clonazepam & 1 \\
\hline
\end{tabular}


Quadro 1 - Características gerais da paciente (Conclusão)

\section{Características demográficas e antropométricas}

\begin{tabular}{lr}
\hline Tratamento medicamentoso & (mg/dia) \\
\hline $\begin{array}{l}\text { Levodopa + Cloridrato de } \\
\text { benserazida }\end{array}$ & 125 \\
\hline
\end{tabular}

Legenda: * = Atividade da vida diária, avaliada por meio da escala de Schwab \& England (10). Essa escala é parte da Unified Parkinson's Disease Rating Scale (UPDRS). A porcentagem das AVDs que 0 indivíduo realiza é, atualmente, assinalada com base no resultado da UPDRS e em relatos do próprio indivíduo ou do cuidador, informando o quanto ele é ou se sente independente para a realização de atividades rotineiras.

Fonte: Dados da pesquisa.

Quadro 2 - Características clínicas da paciente

\begin{tabular}{lr}
\hline Características clínicas & (pontos) \\
\hline UPDRS I (domínio psíquico) & 8 \\
UPDRS II (domínio funcional) & 25 \\
UPDRS III (domínio motor) & 48 \\
UPDRS total & 81 \\
H\&Y & 4 \\
Escala de BERG & 22 \\
MEEM & 16 \\
Teste do Relógio & 4 \\
\hline
\end{tabular}

Legenda: Os testes H\&Y (11, 12), UPDRS (10), Escala de Berg (13), teste do Relógio (14) e MEEM (15) avaliaram, respectivamente, 0 estágio da doença, a severidade da doença, o equilíbrio funcional, as funções executivas e 0 estado mental.

Fonte: Dados da pesquisa.

\section{Protocolo Experimental}

Para avaliação da marcha, a paciente percorreu, andando em sua velocidade preferida, uma passarela de 8 $m$ de comprimento. Para essa tarefa, efetuaram-se quatro tentativas nas seguintes condições: (1) marcha livre, (2) marcha com obstáculo baixo (colocado no centro da passarela e com altura igual a do tornozelo da paciente $-5 \mathrm{~cm}$ ), e (3) marcha com obstáculo alto (altura correspondente à metade da altura do joelho - $20 \mathrm{~cm}$ ). As tentativas foram randomizadas entre as condições.

Para registro dos dados cinemáticos, quatro marcadores passivos reflexivos foram afixados nos seguintes pontos anatômicos: face lateral do calcâneo direito e medial do esquerdo, cabeça do quinto metatarso direito e do primeiro metatarso esquerdo. Uma câmera digital (marca Samsung, modelo SC-D364), com frequência de aquisição de $60 \mathrm{~Hz}$, foi posicionada no plano sagital direito e registrou uma passada completa em cada tentativa, nas três condições.

0 procedimento fotogramétrico foi realizado pelo software Digital Video for Windows (Dvideow Laboratório de Instrumentação em Biomecânica Unicamp, 1998 - versão 6.3). Os dados foram filtrados por um filtro digital Butterworth de segunda ordem $(5 \mathrm{~Hz})$.

\section{Análise dos dados}

As variáveis dependentes, calculadas como em Vitório et al. (16), foram:

a) marcha livre e passada de aproximação dos obstáculos: comprimento do passo (CP), comprimento da passada (CPsd), duração da passada (DPsd), velocidade da passada (VPsd), cadência (CAD), duração da fase de balanço (DFB), duração do suporte simples (DSS), duração do duplo suporte (DDS);

b) marcha com transposição do obstáculo (baixo e alto; Figura 1): distância vertical do pé ao obstáculo do membro de abordagem (DVPOma) e de suporte (DVPOms), distância horizontal do pé ao obstáculo do membro de abordagem (DHPOma) e de suporte (DHPOms), distância horizontal do obstáculo ao pé do membro de abordagem (DHOPma) e comprimento do passo de ultrapassagem (CPultrap).

Os dados foram analisados de forma descritiva (média e desvio padrão).

\section{Resultados}

As variáveis espaciais e temporais da marcha livre, na aproximação do obstáculo baixo e na aproximação do obstáculo alto mostraram resultados bastante 
semelhantes. Entretanto, diminuição espacial foi observada da passada livre para a passada antes do obstáculo alto. 0 mesmo comportamento é visto na velocidade da passada (Quadro 3).

Em relação às fases da marcha, ocorreu aumento progressivo na fase do duplo suporte da passada livre para a passada antes do obstáculo alto (Figura 2).
Durante a transposição do obstáculo, três tentativas foram descartadas por tropeço: uma na transposição do obstáculo baixo e duas na transposição do obstáculo alto. Para as tentativas válidas, para as distâncias horizontais e verticais do pé com o obstáculo, foram observados valores semelhantes para ambas as alturas de obstáculos (Figura 3).

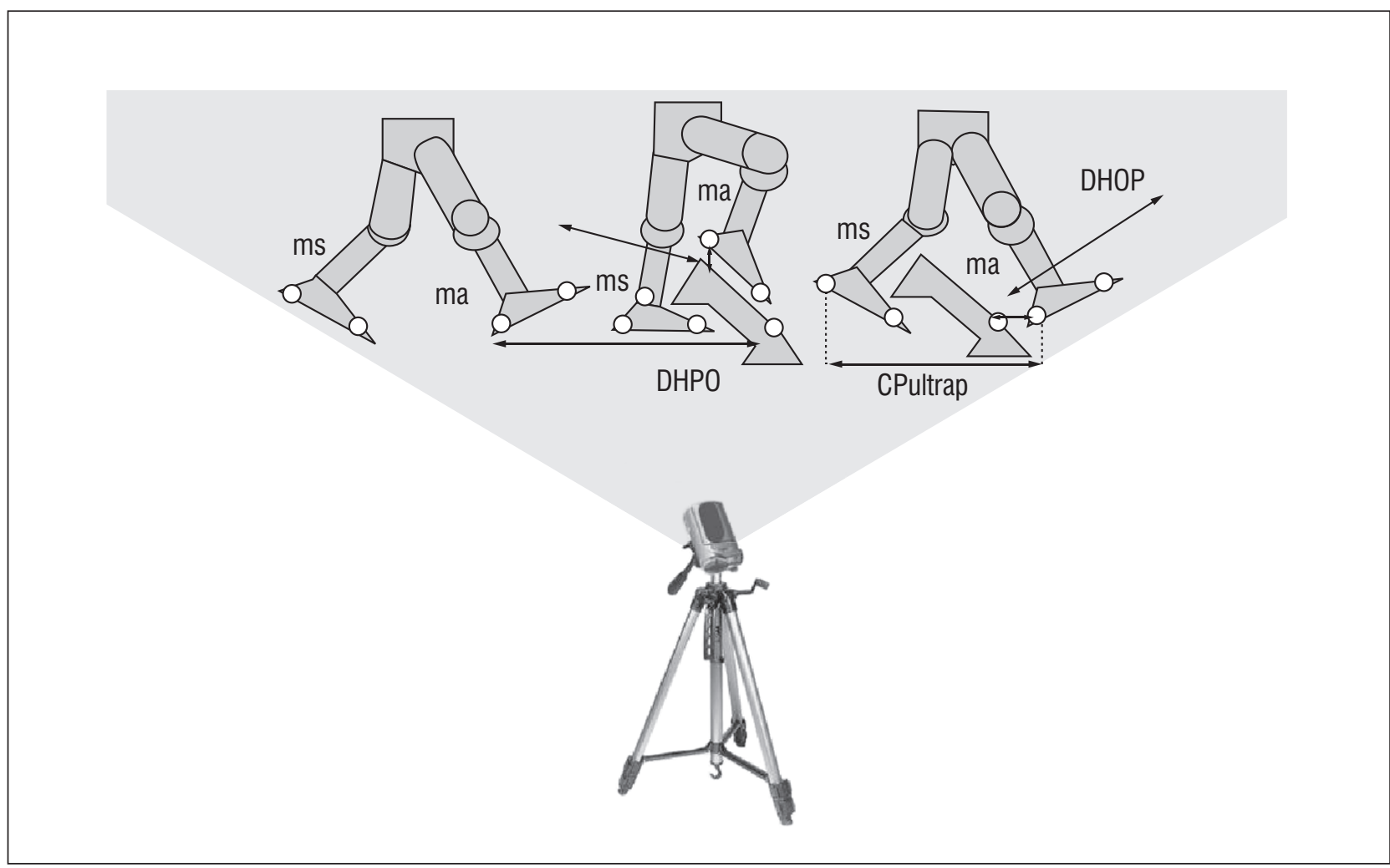

Figura 1 - Esquema representativo da situação experimental e das variáveis dependentes nas condições com obstáculo, distância horizontal pé/obstáculo (DHPO), distância horizontal obstáculo/pé (DHOP), do membro de abordagem (ma) e do membro de suporte (ms), e comprimento do passo de ultrapassagem (CPultrap)

Fonte: Dados da pesquisa.

Quadro 3 - Médias e desvios padrão das variáveis espaciais e temporais da marcha livre e da fase de aproximação do obstáculo baixo e alto

\begin{tabular}{lccc}
\hline Variáveis & Marcha Livre & Obs. Baixo & Obs. Alto \\
\hline Comprimento do passo $(\mathbf{m})$ & $0,37 \pm 0,07$ & $0,30 \pm 0,07$ & $0,26 \pm 0,06$ \\
Comprimento da passada $(\mathbf{m})$ & $0,71 \pm 0,11$ & $0,58 \pm 0,15$ & $0,47 \pm 0,07$ \\
Duração da passada (s) & $1,33 \pm 0,17$ & $1,34 \pm 0,18$ & $1,35 \pm 0,25$ \\
Velocidade da passada (m/s) & $0,55 \pm 0,14$ & $0,43 \pm 0,11$ & $0,36 \pm 0,11$ \\
Cadência (pass/s) & $0,76 \pm 0,09$ & $0,76 \pm 0,10$ & $0,76 \pm 0,14$ \\
\hline
\end{tabular}

Fonte: Dados da pesquisa. 


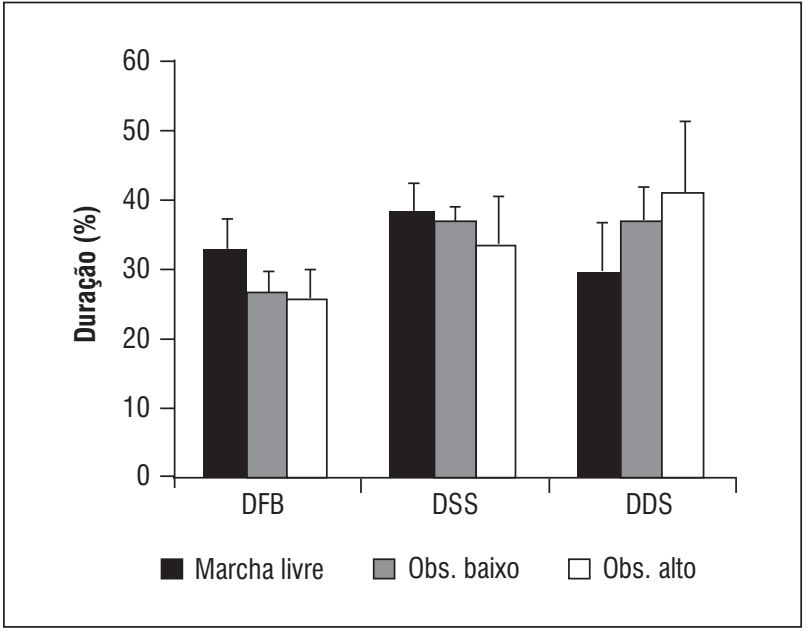

Figura 2 - Médias e desvios padrão da duração da fase de balanço (DFB), duração do suporte simples (DSS) e duração do duplo suporte (DDS) nas condições de marcha livre e na fase de aproximação do obstáculo baixo e do obstáculo alto

Fonte: Dados da pesquisa.

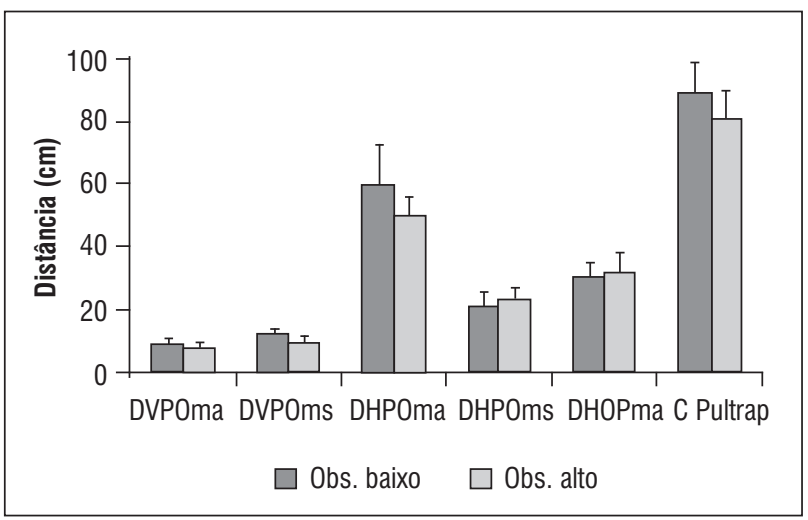

Figura 3 - Médias e desvios padrão da distância vertical pé/ obstáculo (DVPO), distância horizontal pé/obstáculo (DHPO), distância horizontal obstáculo/pé (DHOP), do membro de abordagem (ma) e do membro de suporte (ms) e comprimento do passo de ultrapassagem (CPultrap), nas condições de obstáculo

Fonte: Dados da pesquisa.

\section{Discussão}

Este estudo de caso objetivou descrever o comportamento locomotor de uma paciente com PSP, nas condições de marcha livre e marcha adaptativa. Nas avaliações clínicas, a participante do presente estudo mostrou severidade moderada da doença nos domínios funcional e motor da UPDRS (10), e estágio avançado da doença (4 pontos na escala de H\&Y) $(11,12)$. Neste caso, considerando o tempo de duração da doença (cinco anos), pode-se dizer que a evolução foi rápida a partir dos primeiros sinais e sintomas. A severidade da doença, caracterizada pela escala de H\&Y e pela UPDRS, explica outras características da paciente como a baixa pontuação na escala de equilíbrio funcional de Berg e nas funções cognitivas (MEEM e Teste do Relógio). Em relação ao equilíbrio funcional, 45 pontos é a nota de corte do Teste de Berg para que o indivíduo seja considerado como potencial caidor (13). Nesse caso, a paciente do presente estudo mostra alto grau de instabilidade funcional e risco de quedas. Além disso, a nota de corte no MEEM para indivíduos com escolaridade acima de 11 anos é de 29 pontos (15), indicando que a participante do nosso estudo, com 16 pontos no MEEM e formação superior (14 anos de escolaridade), apresenta acentuado declínio cognitivo.

A baixa pontuação no Teste do Relógio indica que existe comprometimento nas funções executivas, responsáveis pelo planejamento e resolução de problemas (17), componentes que são requisitados na negociação com o obstáculo. Nesse caso, a associação entre a instabilidade, os deficits cognitivos e os comprometimentos da marcha podem explicar o alto índice de quedas da paciente (oito quedas no último ano) e a falta de independência para a realização das atividades da vida diária. Entretanto, apesar desses comprometimentos e da necessidade de supervisão, a participante foi capaz de realizar as tarefas propostas para análise da marcha.

Os parâmetros da marcha da paciente apresentaram valores reduzidos para velocidade e comprimento da passada, caracterizando acentuada bradicinesia e hipometria. Ainda, aumento do duplo suporte, especialmente na aproximação do obstáculo alto, pode indicar procura por maior estabilidade para garantir a transposição do obstáculo com maior segurança. A diminuição da margem de segurança do pé com o obstáculo no momento da ultrapassagem do obstáculo alto também evidenciou comportamento arriscado conforme maior perturbação do ambiente. Comprometimentos na marcha de pacientes com PSP têm sido observados e descritos na literatura $(1,4,6)$. Entretanto, não foram encontrados estudos que descrevessem diretamente os parâmetros da marcha desses pacientes, especialmente em ambientes complexos. Assim, este estudo procurou descrever 
detalhadamente esses parâmetros em ambiente regular e na presença de obstáculos.

Os valores de cadência do presente estudo estão abaixo dos valores descritos na literatura para idosos sadios (ao redor de 100-110 passos/min) $(18,19)$. A velocidade da passada e o comprimento da passada também estão abaixo dos valores encontrados em outros estudos envolvendo idosos sadios $(1,19$ $\pm 0,11 \mathrm{~m} / \mathrm{s} ; 1,24 \pm 0,10 \mathrm{~m}$ ) (8) e pacientes com doença de Parkinson $(0,94 \pm 0,21 \mathrm{~m} / \mathrm{s} ; 1,03 \pm 0,16 \mathrm{~m})$ (8). 0 valor médio da velocidade da paciente com PSP foi de $0,55 \mathrm{~m} / \mathrm{s}$ para a passada livre; no entanto, com o aumento da complexidade do percurso, houve diminuição progressiva dessa velocidade $(0,43 \mathrm{~m} / \mathrm{s}$ na aproximação do obstáculo baixo e $0,36 \mathrm{~m} / \mathrm{s}$ na aproximação do obstáculo alto). Em ambientes complexos (restritos em largura e altura), pacientes com doença de Parkinson apresentam diminuição da velocidade; no entanto, os valores mais baixos desses estudos (20) ainda estão acima dos observados no presente estudo. Em pacientes com doença de Parkinson, os valores encontrados na literatura para o comprimento da passada são de aproximadamente $1,03 \mathrm{~m}$ na marcha livre $(8,20)$; já em condição de restrição do ambiente, esses valores chegam a 0,8 m (20) contra $0,71 \mathrm{~m}, 0,58 \mathrm{~m}$ e $0,47 \mathrm{~m}$ observados em cada condição proposta.

Os estudos acima citados $(8,18-20)$ apresentam resultados de amostras mistas (homens e mulheres). Entretanto, diferenças entre gêneros têm sido observadas nos parâmetros da marcha $(21,22)$. Em adultos jovens, não foram observadas diferenças entre gêneros nas fases de apoio e de duplo suporte. $\mathrm{Na}$ comparação entre gêneros, as mulheres apresentam cadência aumentada e os homens apresentam maior velocidade e comprimento da passada (21). Nesse caso, mesmo utilizando como referência os parâmetros da marcha de mulheres, os resultados da participante do presente estudo se mostram muito abaixo dos encontrados na literatura. Embora não tenham sido encontrados na literatura valores dos parâmetros da marcha de mulheres idosas com PSP ou outro tipo de desordens do movimento, os resultados do presente estudo evidenciam que a paciente estudada apresenta comprometimento da locomoção com acentuada lentidão e hipometria dos movimentos.

Parâmetros rítmicos da marcha como a duração da passada e a cadência podem ser menos afetados pela doença de Parkinson $(7,18)$, o que também foi observado na paciente com PSP. Os valores de cadência e duração da passada se mantiveram constantes mesmo na aproximação dos obstáculos. Ainda, a cadência foi semelhante à observada em idosos sadios (23), sugerindo que o controle desse parâmetro está preservado na paciente estudada.

Nas subfases do ciclo da marcha, as fases de suporte simples e balanço apresentaram resultados similares nas condições de marcha livre e aproximação do obstáculo. No entanto, houve aumento percentual na duração do duplo suporte na fase de aproximação diretamente proporcional à altura do obstáculo. Valores de duração do duplo suporte de $25,6 \%$ foram encontrados para indivíduos com doença de Parkinson e $22,9 \%$ para adultos sadios durante a marcha livre (24). Cabe esclarecer que, para realizar a tarefa com menor risco e custo energético, a locomoção necessita do acoplamento da informação sensorial e da modulação do sistema efetor $(25,26)$; assim, qualquer degeneração dos componentes ou subsistemas pode dificultar a realização da tarefa (27), o que parece ocorrer na PSP. Entretanto, o aumento na duração do duplo suporte na fase de aproximação do obstáculo alto sugere tentativa de modulação do movimento na condição de maior restrição do ambiente. Esse comportamento sugere que, apesar dos comprometimentos evidentes na marcha, o sistema ainda é capaz de modular os parâmetros da marcha nesta fase do percurso $(27,28)$.

Desviar ou transpor um obstáculo exige adaptação constante da locomoção $(26,27)$. Ainda, a ação motora de ultrapassar obstáculos apresenta um risco potencial de tropeço e queda (9). Os resultados similares das variáveis de ultrapassagem nas duas condições sugerem que:

a) o obstáculo alto não foi suficientemente desafiador a ponto de exigir alterações nos padrões previamente adotados na ultrapassagem do obstáculo baixo;

b) os comprometimentos resultantes da PSP (paralisia do olhar, instabilidade postural e deficits cognitivos) observados na paciente estudada implicaram a percepção das informações sensoriais e na resposta do sistema efetor, não permitindo ajustes eficientes do padrão locomotor em função da altura do obstáculo.

Durante a coleta de dados, na marcha com obstáculos, algumas tentativas foram descartadas por tropeços, principalmente diante do obstáculo alto, 
o que indica que a hipótese (a) não é verdadeira. Observou-se perturbação, uma vez que houve dificuldade e eventuais insucessos na realização da tarefa. Assim, infere-se que a similaridade nos parâmetros da marcha durante a transposição dos obstáculos foi consequência da rigidez do sistema para adequar o movimento conforme maior perturbação do ambiente (obstáculo alto). Dessa forma, embora ajustes tenham sido observados, as modulações não foram suficientes para garantir o sucesso em todas as tentativas, especialmente na transposição do obstáculo alto.

A doença de Parkinson e a PSP têm sido classificadas como desordens do movimento com comprometimento nos núcleos da base. Pacientes com doença de Parkinson nos estágios iniciais e moderados apresentam preservação da capacidade de modular o sistema efetor de acordo com as demandas do ambiente $(16,20)$, o que não foi observado na paciente com PSP. Apesar de também haver comprometimento nos núcleos da base, os núcleos cerebelares e o tronco cerebral também estão em processo degenerativo na PSP (2).

Os parâmetros espaciais de transposição do obstáculo refletem modulação do sistema efetor a partir do julgamento perceptivo da localização e dimensão do obstáculo $(9,29)$. Para garantir segurança e estabilidade, a modulação ocorre na tomada de decisões durante a aproximação do obstáculo imediatamente antes de ultrapassá-lo (distância horizontal pé/obstáculo), na margem de segurança sobre o obstáculo (distância vertical pé/obstáculo) e no momento da aterrissagem do pé após a ultrapassagem (distância horizontal obstáculo/pé) $(9,30)$. As distâncias horizontais pé/obstáculo e obstáculo/pé, tanto em pacientes com doença de Parkinson como em idosos sadios, têm se mostrado resistentes à altura do obstáculo $(9,16,30)$, o que ratifica os resultados do presente estudo em paciente com PSP. No entanto, pacientes com doença de Parkinson aumentam a margem de segurança frente ao obstáculo alto (em torno de 11 cm para obstáculo baixo e $14,5 \mathrm{~cm}$ para obstáculo alto) durante a ultrapassagem justamente para reduzir os riscos de tropeços $(9,16)$; diferentemente, a paciente estudada diminuiu a distância vertical pé/obstáculo na presença do obstáculo alto. Esse comportamento arriscado sugere dificuldade para adequar a resposta motora a partir da percepção das características do ambiente.

\section{Conclusão}

A partir deste estudo, nota-se que a PSP afetou de forma evidente a marcha da paciente estudada. Durante a aproximação do obstáculo, algumas estratégias foram observadas, como diminuição discreta no comprimento e na velocidade da passada e aumento da duração do duplo suporte. No entanto, durante a negociação com os obstáculos, foi evidente a deficiência do sistema em adotar estratégia eficiente para a realização da tarefa. Nas condições de obstáculo, os parâmetros avaliados não sofreram grandes modulações apesar do aumento na altura do obstáculo. Essa inflexibilidade para a adaptação da marcha ao ambiente pode aumentar o risco de tropeços e quedas durante a locomoção em ambientes complexos.

Estudos com amostras maiores devem ser realizados para que esses resultados possam ser generalizados. Por ora, é possível sugerir que, embora pacientes com PSP apresentem parkinsonismo, observando-se similaridades entre as doenças, programas de exercício físico e de reabilitação para estes pacientes sejam diferenciados o intento de atender às características locomotoras específicas da doença.

\section{Referências}

1. Suteerawattananon M, Macneill B, Protas EJ. Supported treadmill training for gait and balance in a patient with progressive supranuclear palsy. Phys Ther. 2002;82(5):485-95. PMid:1199180.

2. Nath U, Ben-Shlomo Y, Thomson RG, Lees AJ, Burn DJ. Clinical features and natural history of progressive supranuclear palsy: a clinical cohort study. Neurology. 2003;60(2):910-6. doi:10.1212/01. WNL.0000052991.70149.68.

3. Carrilho PE, Barbosa ER. Progressive supranuclear palsy in a sample of brazilian population: clinical features of 16 patients. Arq Neuropsiquiatr. 2002;60:91722. doi:10.1590/S0004-282X2002000600006.

4. Rosa PA, Ferreira CR. Degeneração Walleriana de origem vascular em ressonância magnética de paciente com paralisia supranuclear progressiva provável. Arq Neuropsiquiatr. 2005;63(3B):878-80. doi:10.1590/ S0004-282X2005000500032. 
5. Uttl B, Santacruz P, Litvan I, Grafman J. Caregiving in progressive supranuclear palsy. Neurology. 1998; 51:1303-9. doi:10.1212/WNL.51.5.1303.

6. Di Fabio RP, Zampieri C, Tuite P. Gaze control and foot kinematics during stair climbing: characteristics leading to fall risk in progressive supranuclear palsy. Phys Ther. 2008;88:240-50. doi:10.2522/ptj.20070159.

7. Morris ME, Iansek R, Matyas TA, Summers JJ. Ability to modulate walking cadence remains intact in Parkinson's disease. J Neurol Neurosurg Psychiatry. 1994;57(12):1532-4. doi:10.1136/jnnp.57.12.1532.

8. Sofuwa O, Nieuwboer A, Desloovere K, Willems AM, Chavret F, Jonkers I. Quantitative gait analysis in Parkinson's disease: comparison with a healthy control group. Arch Phys Med Rehabil. 2005;86(5):1007-13. doi:10.1016/j.apmr.2004.08.012.

9. Pieruccini-Faria F, Menuchi MP, Vitorio R, Gobbi LTB, Stella F, Gobbi S. Parâmetros cinemáticos da marcha com obstáculos em idosos com Doença de Parkinson, com e sem efeito da levodopa: um estudo piloto. Rev Bras Fisioter. 2006;10(2):233-9. doi:10.1590/ S1413-35552006000200015.

10. Fahn S, Elton R. Members of the UPDRS. Development Comitee. The unified Parkinson's disease rating scale. In: Fahn S, Marsden CD, Calne DB, Goldstein M, editors. Recent developments in Parkinson's disease. vol. 2. Florham Park: Mcmellam Health Care Information; 1987. p.153-63.

11. Hoehn MM, Yahr MD. Parkinsonism: onset, progression and mortality. Neurology. 1967;7:427-42. doi:10.1212/WNL.17.5.427.

12. Goetz CG, Poewe W, Rascol O, Sampaio C, Stebbins GT, Counsell C, et al. Movement disorder society task force report on the Hoehn and Yahr staging scale: status and recommendations. Mov Disord. 2004;19(9):1020-28. doi:10.1002/mds.20213.

13. Miyamoto ST, Lombardi J, Berg KO, Ramos LR, Natour J. Brazilian version of the berg balance scale. Braz J Med Biol Res. 2004;37:1411-21. doi:10.1590/ S0100-879X2004000900017.

14. Berger G, Frölich L, Weber B, Pantel J. Dementia diagnostic accuracy of the clock drawing test: the relevance of "time setting" in screening for dementia. J Geriatr Psychiatry Neurol. 2008;21:250-60. PMid:19017782.
15. Brucki SMD, Nitrini R, Caramelli P, Bertolucci PHF, Okamoto IH. Sugestões para o uso do Mini-exame do Estado Mental no Brasil. Arq Neuropsiq. 2003;61(3): 777-81. doi:10.1590/S0004-282X2003000500014.

16. Vitório R, Pieruccini-Faria F, Stella F, Gobbi S, Gobbi LTB. Effects of obstacle height on obstacle crossing in mild Parkinson's disease. Gait Posture. 2010;31:1436. doi:10.1016/j.gaitpost.2009.09.011.

17. Lezak MD. Neuropsychological Assessment. 3. ed. New York: Oxford University Press; 1995.

18. Blin O, Ferrandez AM, Serratrice G. Quantitative analysis of gait in Parkinson's patients: increased variability in stride length. J Neurol Sci. 1990;98(1):91-7. doi:10.1016/0022-510X(90)90184-0.

19. O'Sullivan JD, Said CM, Dillon LC, Hoffman M, Hughes AJ. Gait analysis in patients with Parkinson's disease and motor fluctuations: influence of levodopa and comparison with other measures of motor function. Mov Disord. 1998;13:900-6. doi:10.1002/ mds.870130607.

20. Caetano MJD, Gobbi LTB, Sánchez-Arias MR, Stella F, Gobbi S. Effects of postural threat on walking features of Parkinson's disease patients. Neurosci Lett. 2009;452:136-40. doi:10.1016/j.neulet. 2009. 01.053.

21. Cho SH, Park JM, Kwon OY. Gender differences in three dimensional gait analysis data from 98 healthy Korean Adults. Clin Biomech. 2004;19(2):145-52. doi:10.1016/j.clinbiomech.2003.10.003.

22. Kerrigan DC, Todd MK, Della Croce U. Gender differences in joint biomechanics during walking: normative study in young adults. Am J Phys Med Rehabilit. 1998, 77(1):2-7. doi:10.1097/00002060-199801000-00002.

23. Crenna P, Carpinella I, Rabuffetti M, Calabrese E, Mazzoleni P, Nemni R, et al. The association between impaired turning and normal straight walking in Parkinson's disease. Gait Posture. 2007;26(2):172-8. doi:10.1016/j.gaitpost.2007.04.010.

24. Baltadjieva R, Giladi N, Gruendlinger L, Peretz C, Hausdorff JM. Marked alterations in the gait timing and rhythmicity of patients with de novo Parkinson's disease. Eur J Neurosci. 2006;24:181520. doi:10.1111/j.1460-9568.2006.05033.x. 
25. Gobbi LTB, Patla A. Desenvolvimento da locomoção em terrenos irregulares: proposta de um modelo teórico. In: Pellegrini AM, organizador. Coletânea de estudos: comportamento motor I. São Paulo: Movimento; 1997. p. 29-44.

26. Moraes R, Gobbi LTB. Controle visual da locomoção adaptativa e mobilidade em indivíduos idosos. In: U. Corrêa, editor. Pesquisa em comportamento motor: a intervenção profissional em perspectiva. São Paulo: EFP/EEFEUSP; 2008. p.42-60.

27. Gobbi LTB, Pieruccini-Faria F, Silveira CRA, Caetano MJD. Núcleos da base e controle locomotor: aspectos neurofisiológicos e evidências experimentais. Rev Bras Educ Fís Esp. 2006;20(Supl.5):97-101.

28. Grillner S, Hellgren J, Ménard A, Saitoh K, Wikström MA. Mechanisms for selection of basic motor programs: roles for the striatum and pallidum. Trends Neurosci. 2005;28(7):364-70. doi:10.1016/j.tins.2005.05.004.
29. Chen HC, Ashton-Miller JA, Alexander NR, Schultz AR. Stepping over obstacles: gait patterns of healthy young and old adults. J Gerontol. 1991;46(6):M196203. doi:10.1093/geronj/46.6.M196.

30. Sparrow WA, Shinkfield AJ, Chow S, Begg RK. Characteristics of gait in stepping over obstacles. Hum Mov Sci. 1996;15:605-22. doi:10.1016/ 0167-9457(96)00022-X.

Recebido: $11 / 05 / 2011$

Received: 05/11/2011

Aprovado: 09/01/2012

Approved: 01/09/2012 